\title{
Hurgando en el arcón de los tiempos perdidos en los confines de la ecúmene. Las etnias selk'nam, yámana y halakwulup según el registro somatométrico de Martín Gusinde (1989 [1937])
}

\author{
José A. Cocilovo, Héctor H. Varela y Silvia G. Valdano \\ Recibido 01 de octubre 2018. Aceptado 28 de diciembre 2018
}

\begin{abstract}
RESUMEN
Durante el siglo pasado, investigaciones realizadas sobre las etnias del extremo austral del continente americano proporcionaron abundante información sobre la etnografía y las características físicas que reflejan la composición de los fenotipos locales relacionados con selk'nam, yámana y halakwulup, entre las que se destaca la contribución de Martín Gusinde. En este trabajo se explora la estructura de la población nativa y las relaciones de parentesco sobre la base de rasgos fenotípicos con la evaluación de 18 variables cefalométricas en 110 individuos. El análisis estadístico multivariado confirma la existencia de diferencias significativas entre grupos y entre sexos por grupos. Se comprobó una marcada estructura de la población y un balance diferente entre grupos con relación a los efectos de la deriva genética y la migración. Halakwulup presentó una varianza fenotípica mayor que la esperada comparada con selk'nam y yámana. Las varianzas observadas de ambos sexos halakwulup y hombres selk'nam revelan su interacción en un circuito territorial mayor, mientras que las varianzas de ambos sexos yámana y mujeres selk'nam indican menor tamaño efectivo y su interacción en un ámbito más restringido. Se destaca el valor de la información recuperada pues refleja la condición humana en el límite de la supervivencia después de 10.000 años de historia.
\end{abstract}

Palabras clave: Relaciones genéticas; Dimorfismo sexual; Estructura territorial.

\begin{abstract}
RUMMAGING IN THE CHEST OF LOST TIME AT THE EDGES OF THE INHABITED WORLD: THE SELK'NAM, YÁMANA AND HALAKWULUP ETHNIC GROUPS ACCORDING TO GUSINDE'S (1989 [1937]) SOMATOMETRY. During twentieth century, research on ethnic groups in the far south of the American continent provided much information on the ethnography and physical characteristics that reflect the composition of local phenotypes related to the Selk'nam, Yámana and Halakwulup, in which the contribution of Martín Gusinde stands out. This paper explores the structure of the native population and kinship relationships based on phenotypic traits resulting from the evaluation of 18 cephalometric variables in 110 individuals. Multivariate statistical analysis confirms the existence of significant differences between groups and between sexes by group. A marked population structure and a balance between genetic drift and different migration between groups were found. Halakwulup presented a higher phenotypic variance
\end{abstract}

José A. Cocilovo. Consejo Nacional de Investigaciones Científicas y Técnicas (CONICET). Departamento de Ciencias Naturales, Facultad de Ciencias Exactas, Físico-Químicas y Naturales, Universidad Nacional de Río Cuarto (UNRC). Ruta 36 km 601 (5800), Río Cuarto (Córdoba, Argentina). E-mail: jcocilovo@exa.unrc.edu.ar

Héctor H. Varela. CONICET. Departamento de Ciencias Naturales, Facultad de Ciencias Exactas, Físico-Químicas y Naturales, Universidad Nacional de Río Cuarto (UNRC). Ruta 36 km 601 (5800), Río Cuarto (Córdoba, Argentina) E-mail: hvarela@exa.unrc.edu.ar

Silvia G. Valdano. CONICET. Departamento de Ciencias Naturales, Facultad de Ciencias Exactas, Físico-Químicas y Naturales, Universidad Nacional de Río Cuarto (UNRC). Ruta $36 \mathrm{~km} 601$ (5800), Río Cuarto (Córdoba, Argentina). E-mail: svaldano@exa.unrc.edu.ar 
than expected compared with Selk'nam and Yámana. The observed variances of both sexes among the Halakwulup and Selk'nam men reveal their interaction in a larger territorial circuit, while the variances of both sexes among the Yámana and Selk'nam women indicate a smaller effective size and their interaction in a more restricted environment. The value of the study is that it highlights the human condition at the limit of survival after 10,000 years of history.

Keywords: Genetic relationships; Sexual dimorphism; Territorial structure.

\section{INTRODUCCIÓN}

La Isla Grande de Tierra del Fuego ocupa un territorio de $47.992 \mathrm{~km}^{2}$ y, junto con el archipiélago circundante, alcanza una superficie de $73.753 \mathrm{~km}^{2}$ (Figura 1). El poblamiento del territorio más austral de Sudamérica se produjo entre 13.500 y 10.500 AP por grupos de cazadores-recolectores de adaptación continental y marítima (Miotti y Salemme 2004). En el archipiélago occidental de las islas y canales fueguinos se determinó la ocupación desde inicios del Holoceno medio (9000-7000 AP) (Ocampo y Rivas 2004). Para la costa norte y noroccidental de Tierra del Fuego se obtuvieron fechas iniciales de entre 5565 y $1460 \pm 40$ AP (Morello et al. 2009, 2012).

En el siglo XVI se produjo el primer encuentro entre europeos y las poblaciones aborígenes de Tierra del Fuego en Puerto San Julián, por la expedición española que en 1520, al mando de Fernando de Magallanes, descubrió el estrecho que permitía la conexión entre el Atlántico y el Pacífico. La población nativa estaba representada por tres etnias principales: los selk'nam, de elevada talla y musculatura levemente acentuada; los yámana, de menor estatura y un mayor desarrollo de las extremidades superiores con respecto a los primeros; $y$ los halakwulup, con un esqueleto fuerte, hombros anchos y una relación armónica entre la talla, el tronco y los miembros (Gusinde 1989 [1937]).

Esas definiciones realizadas por Gusinde sugirieron una hipótesis útil que luego fue probada empleando la información somatométrica disponible en su obra. El análisis estadístico permitió caracterizar a los grupos de Tierra del Fuego por las diferencias significativas entre las etnias selk'nam, yámana y halakwulup y sustentar una teoría sobre el poblamiento del archipiélago a partir de corrientes migratorias provenientes de los territorios del oriente y el occidente de la cordillera andina (Guichón et

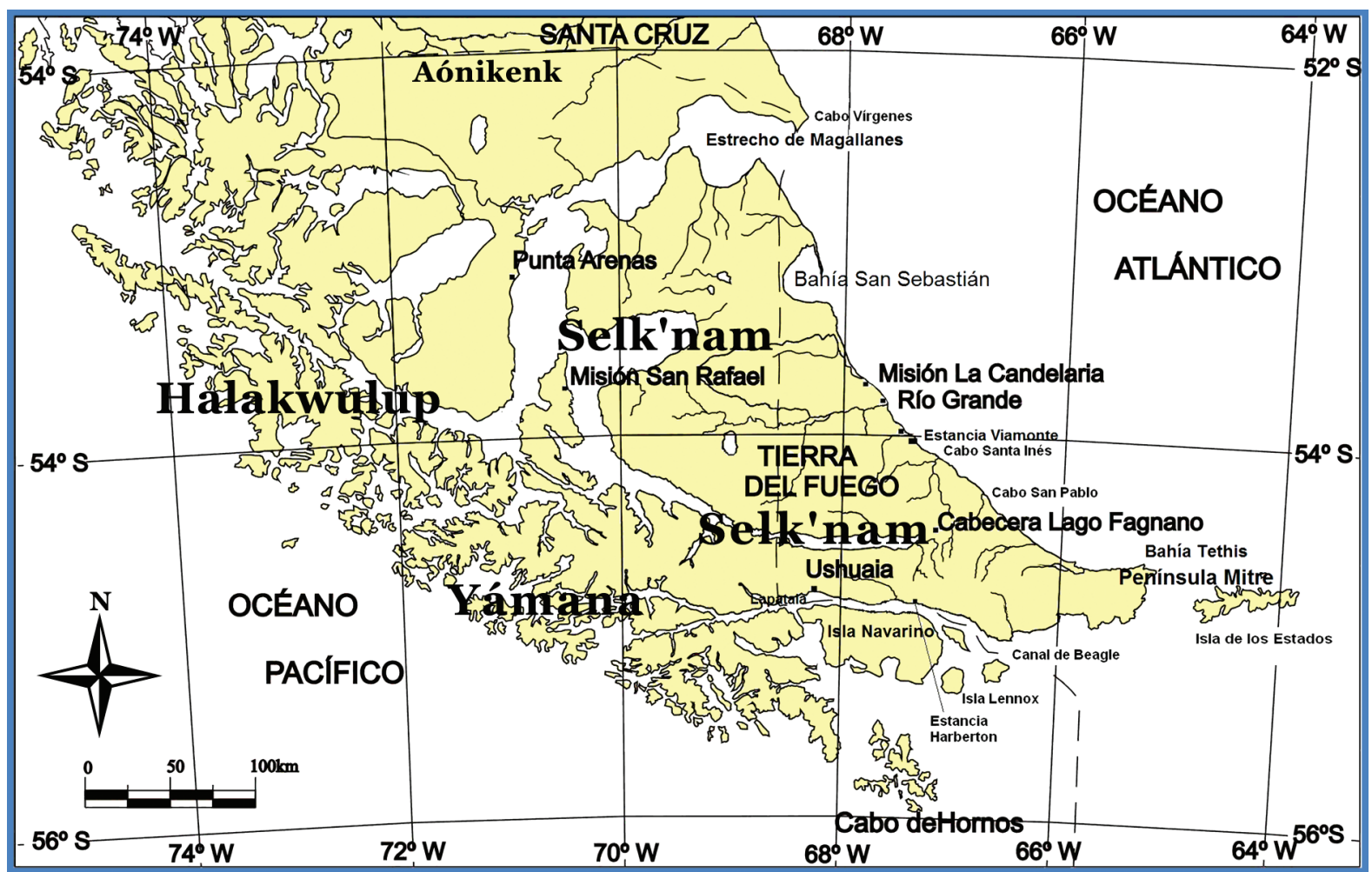

Figura 1. Tierra del Fuego. Áreas aproximadas de asentamientos selk'nam, yámana y halakwulup (modificado a partir de Casali 2013, Fig. 1). 
al. 1989-1990; Varela et al. 1993-1994). Además, se realizaron análisis completos de las bases de restos antiguos (Cocilovo y Guichón 1985-1986, 19992000) y modernos obtenidas por Gusinde sobre las tres etnias mencionadas (Varela et al. 1993-1994, 1997).

Si bien los análisis realizados destacaban la existencia de grupos fenotípicamente diferentes y sugerían posibles relaciones, con el diseño estadístico empleado no fue posible realizar inferencias sobre el parentesco entre los individuos de cada grupo ni entre los miembros de los diferentes grupos, y tampoco evaluar el proceso evolutivo a partir del equilibrio entre la deriva genética y la migración. Por esta razón, en el presente trabajo se analiza la estructura de la población, considerando las relaciones de parentesco anotadas en la obra citada (Gusinde 1989 [1937]). Tales relaciones tienen que haber experimentado los avatares de la colonización inicial y, posteriormente, el impacto de la conquista y colonización europea. Se espera que los resultados del análisis realizado reflejen las condiciones de la supervivencia de la población en el límite de la extinción.

\section{MATERIAL Y MÉTODOS}

Para la realización del presente trabajo se empleó una muestra de 110 individuos de las etnias selk'nam, yámana y halakwulup de ambos sexos, de edades juvenil, adulto, maduro y senil, obtenida por Gusinde (1989 [1937]) entre 1919 y 1924. Se emplearon 18 variables somatométricas, que se mencionan en la Tabla 1.

\section{Referencias sobre las etnias según Gusinde (1989 [1937])}

La etnia selk'nam estaba conformada por cazadores nómades que habitaban en la Isla Grande de Tierra del Fuego. Con relación a este grupo, se mencionan tres parcialidades: 1) p'áriska: gente del

\begin{tabular}{|l|l|}
\hline Talla total & Altura facial fisionómica \\
\hline Longitud miembro superior & Altura facial morfológica \\
\hline Longitud miembro inferior & Altura facial superior morfológica \\
\hline Longitud cefálica máxima & Perímetro cefálico horizontal \\
\hline Anchura cefálica máxima & Arco cefálico sagital \\
\hline Anchura biauricular & Arco cefálico transversal \\
\hline Anchura bicigomática & Altura de la nariz \\
\hline Anchura bigoníaca & Longitud de la nariz \\
\hline Altura de la cabeza auricular & Anchura base nasal \\
\hline
\end{tabular}

Tabla 1. Variables somatométricas. Denominación. norte de Río Grande; 2) háriska: gente de los bosques del sur, de la región montañosa y 3) winteka (haus); en península Mitre. Se estimaba la población inicial en 3500-4000 personas (1989 [1937], Tomo 4: 7) hasta 1880, cuando se puso en marcha el exterminio a manos europeas; en 1919 (primer viaje de Gusinde), la población era de 276 individuos (Tomo 4: 11). Sin embargo, estimaciones modernas proponen, en el momento de contacto con españoles, la cifra más probable de 1500 personas, con una densidad de 0,04 personas $/ \mathrm{km}^{2}$ y 37 integrantes de cada territorio familiar (haruwen) (García-Moro et al. 1997).

Los yámana habitaban en el archipiélago del cabo de Hornos en la costa norte del canal de Beagle; eran canoeros, dedicados a la caza de aves, león y/o lobo marino, y a la recolección de moluscos y productos vegetales. La población inicial se calculaba en 2500 personas, la cual, para 1919 no llegaba a los 80 individuos. Se comprobó una rápida extinción y, hacia 1938, quedaban 24 individuos (Tomo 4: 16).

Los halakwulup habitaban el territorio definido como "Mundo Insular Patagónico Occidental", canales de Patagonia occidental, islas e islotes entre la península Brecknock en el sur y el canal Messier en el golfo de Penas al norte. Se mencionan tres grupos: sur, central y norte (Tomo 4: 18). Eran canoeros nómades similares a los yámana, dedicados a la pesca, caza y recolección. Integraban grupos familiares aislados. La población original se calculaba en 5000 personas, y en 1924 quedaban solo 245-250 individuos (Tomo 4: 22).

A partir de la lectura de la obra citada se sintetizan algunas referencias sobre las muestras que permiten evaluar la naturaleza de estas y los resultados de la presente experiencia. El grupo selk'nam fue integrado con 24 hombres y 22 mujeres; se destaca un caso de parentesco filial y se incluye un individuo haus. Se mencionan elevada fecundidad y mortalidad infantil. Además, Gusinde declara la práctica de la exogamia y refiere algunos casos de apareamiento con europeos (Tomo 4: 65). Con relación a yámana, la muestra obtenida fue integrada con 14 hombres y 19 mujeres. Se declara la existencia de individuos emparentados directamente (progenitor-descendiente) y con individuos de la etnia selk'nam por matrimonio doble (Tomo 4: 76-77).

La muestra halakwulup proviene de un amplio territorio: Puerto Harris, isla Dawson, Puerto 
Galante, canal Magdalena, entre otros. Se trata de 15 hombres y 16 mujeres examinados en 1923; la tribu se distribuía en tres grupos geográficos y sumaba 250 personas. A fines de 1938 se registraron 120 individuos. Además, se declaran relaciones de parentesco filial, apareamientos con europeos y el impacto de enfermedades venéreas. Gusinde advierte de la naturaleza de la muestra estudiada, sobre su escasez y su "valor intrínseco", y expresa que las mediciones fueron realizadas de acuerdo con los instrumentos recomendados por Martin en su obra Lehrbuch der Anthropologie (Tomo 4: 25).

\section{Análisis de la información somatométrica}

Para evaluar la integración de la muestra se analizó la distribución del sexo y de la edad mediante pruebas $\chi^{2}$. Según las edades registradas en el relevamiento de Gusinde, los individuos fueron agrupados en intervalos de clase. Se analizaron las diferencias fenotípicas entre las etnias y entre sexos por etnia mediante técnicas estadísticas de análisis multivariado de la varianza (MANOVA) y análisis discriminante (Seber 1984). Además, se evaluó la clasificación de los individuos y la de cada sexo por la probabilidad de pertenecer a una u otra etnia (Regla de Bayes, en Montes Suay 2007).

De acuerdo con los antecedentes antes citados y suponiendo que los grupos son el resultado de un mismo proceso evolutivo que durante milenios operó en Patagonia austral, se estimaron valores Fst para rasgos cuantitativos según Relethford y Blangero (1990), Relethford (1994), Relethford y Harpending (1994) y Relethford et al. (1997). El estadístico Fst, en ausencia de efectos mutacionales y selectivos, permite medir la divergencia genética y las relaciones de parentesco entre las poblaciones. Este valor expresa la magnitud de la varianza promedio dentro de grupos con relación a la varianza entre grupos. Esta relación depende del balance entre la deriva genética y la migración, factores evolutivos cuyo equilibrio tiene que haber experimentado cambios durante la conquista y colonización inicial del territorio austral, y tardíamente por el impacto de la ocupación y colonización por europeos. Se empleó el programa RMET versión 5.0 (Relethford 2003) para la estimación del estadístico Fst con una heredabilidad de 0,55 de acuerdo con lo recomendado por Relethford y Blangero (1990) y con las experiencias realizadas (Varela y Cocilovo 1999, 2007; Medeot et al. 2008) en poblaciones locales sobre la varianza genética máxima para caracteres cuantitativos según Falconer y Mackay (1996). Las varianzas residuales que indican la desviación de la varianza observada con respecto a la esperada en el equilibrio deriva/migración se evaluaron por la distribución $t$ de acuerdo con Relethford y Harpending (1994). El análisis supone la equivalencia entre las matrices de varianza genéticas $\left(\mathrm{V}_{\mathrm{G}}\right)$ y las matrices de varianza fenotípicas $\left(\mathrm{V}_{\mathrm{p}}\right)$.

En un determinado espacio geográfico, la deriva disminuye la variación genética dentro de grupos e incrementa la divergencia, mientras que el efecto de la migración tiende a restaurar la variabilidad y aumenta las semejanzas entre los grupos. La estimación del estadístico Fst se realizó entre grupos, entre pares de grupos, entre grupos para cada sexo y entre sexos para cada grupo. En cada caso, se calculó además el flujo génico para evaluar el nivel de aislamiento genético entre grupos a partir del número de individuos migrantes por generación (indmig/gen) mediante la expresión original de Sewall Wright: $\mathrm{Nm}=1 / 4 *[(1 / \mathrm{Fst})-1]$.

\section{RESULTADOS}

En la Tabla 1 se presentan las variables empleadas en el análisis. La muestra está compuesta por $48 \%$ de hombres y $52 \%$ de mujeres (Tabla 2 ) sin diferencias significativas en las proporciones de acuerdo con la prueba $\chi^{2}=0,73(g /=2, p=0,693)$. En la Tabla 3, la distribución por edades tampoco indica diferencias significativas $\left(\chi^{2}=8,83, g l=10\right.$, $p=0,548)$; sin embargo, se observa una proporción mayor de individuos jóvenes en la cohorte de entre 21-30 años en todos los grupos (Figura 2).

\begin{tabular}{|l|c|c|c|}
\hline \multirow{2}{*}{ Grupo } & \multicolumn{2}{|c|}{ Sexo } & \multirow{2}{*}{ Total } \\
\cline { 2 - 3 } & Masculinos & Femeninos & \multirow{2}{*}{ Telk'nam } \\
\hline Yámana & $14(52,2)$ & $22(47,8)$ & 46 \\
Halakwulup & $15(48,4)$ & $19(57,6)$ & 33 \\
\hline Total & $\mathbf{5 3 ( 4 8 , 2 )}$ & $\mathbf{5 7}(\mathbf{5 1 , 8 )}$ & $\mathbf{1 1 0}$ \\
\hline
\end{tabular}

Nota: $\chi^{2}=0,73, g l=2, p=0,693$ (porcentajes por fila entre paréntesis).

Tabla 2. Composición de la muestra.

\begin{tabular}{|c|c|c|c|c|c|c|c|c|c|}
\hline \multirow[b]{3}{*}{ Juveniles } & \multirow{3}{*}{$\frac{\text { Edad }}{18-20}$} & \multicolumn{6}{|c|}{ Grupo } & \multirow{2}{*}{\multicolumn{2}{|c|}{ Total }} \\
\hline & & \multicolumn{2}{|c|}{ Selk'nam } & \multicolumn{2}{|c|}{ Yámana } & \multicolumn{2}{|c|}{ Halakwulup } & & \\
\hline & & 2 & $(4,3)$ & 3 & $(9,1)$ & 1 & $(3,2)$ & 6 & $(5,5)$ \\
\hline \multirow{2}{*}{ Adultos } & $21-30$ & 22 & $(47,8)$ & 10 & $(30,3)$ & 12 & $(38,7)$ & 44 & $(40,0)$ \\
\hline & $31-40$ & 6 & $(13,0)$ & 5 & $(15,2)$ & 7 & $(22,6)$ & 18 & $(16,4)$ \\
\hline Maduros & $41-50$ & 9 & $(19,6)$ & 5 & $(15,2)$ & 3 & $(9,7)$ & 17 & $(15,5)$ \\
\hline \multirow{3}{*}{ Seniles } & $51-60$ & 5 & $(10,9)$ & 6 & $(18,2)$ & 3 & $(9,7)$ & 14 & $(12,7)$ \\
\hline & $61+$ & 2 & $(4,3)$ & 4 & $(12,1)$ & 5 & $(16,1)$ & 11 & $(10,0)$ \\
\hline & Total & \multicolumn{2}{|l|}{46} & \multicolumn{2}{|l|}{33} & \multicolumn{2}{|l|}{31} & \multicolumn{2}{|c|}{110} \\
\hline
\end{tabular}

Nota: $\chi^{2}=8,83, g l=10, p=0,548$ (porcentajes por columna entre paréntesis).

Tabla 3. Distribución de las edades por grupo. 


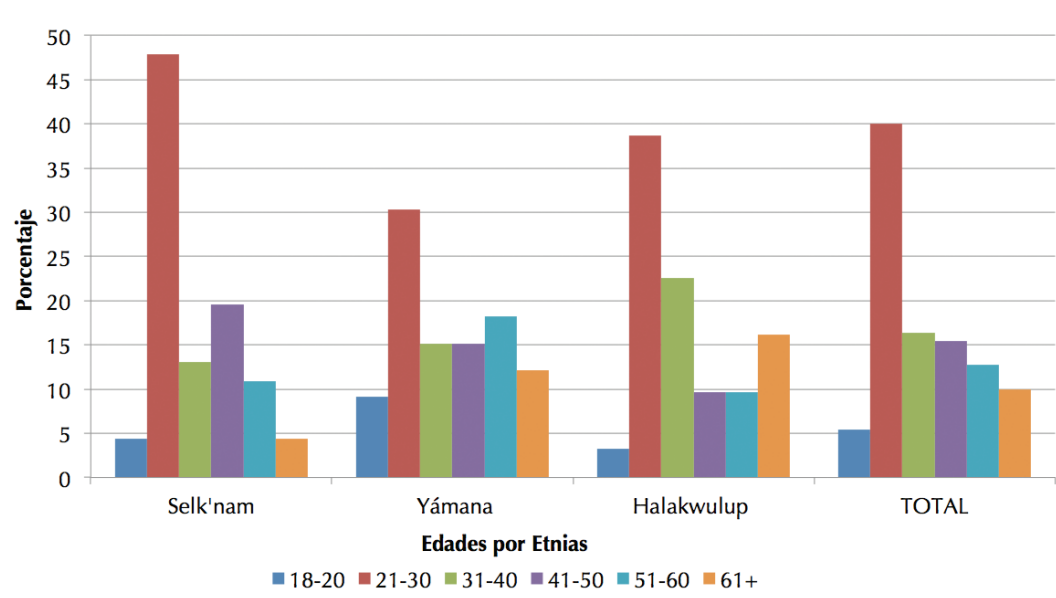

Figura 2. Distribución de los individuos por edad y etnia.

El análisis estadístico multivariado indicó la existencia de diferencias fenotípicas significativas entre etnias: Lambda de Wilks=0,119 aprox. $F_{36,180}=$ $9,52, p<0,001$. La evaluación de los valores $D^{2}$ de Mahalanobis confirma las diferencias entre pares de grupos ( $g l=18$ y 90, $p<0,001$, Figura 3). La re-

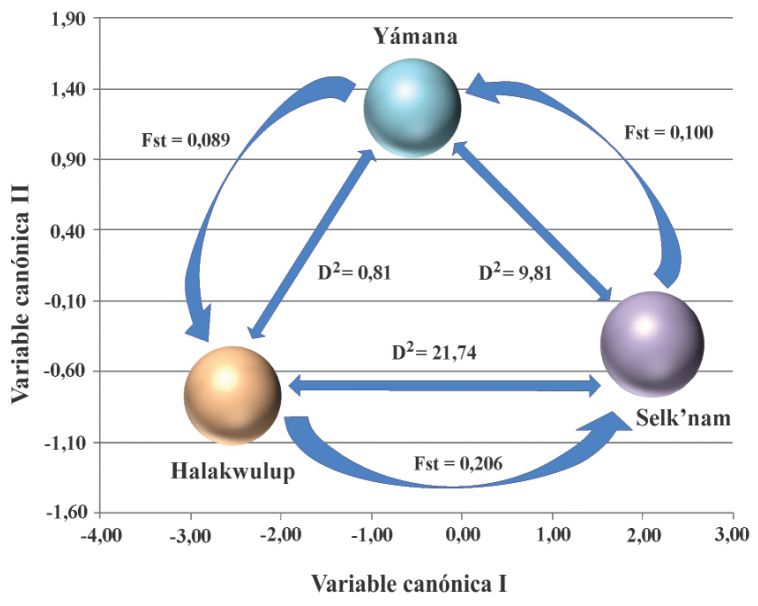

Figura 3. Síntesis sobre la estructura de la población, valores Fst y $D^{2}$ entre etnias. Coordenadas discriminantes I y II.

clasificación de las observaciones por la mayor probabilidad de pertenecer a una u otra etnia se presenta en la Tabla 4. Las asignaciones al propio grupo original alcanzaron al 90\% de los casos en promedio, y el $10 \%$ restante fue clasificado en una etnia diferente. Yámana es el grupo que presenta el mayor número de casos asignados a otros grupos (18\%).

La estructura de sexos entre grupos se presenta sobre la base de los valores $D^{2}$ en la Tabla 5 (todos los valores $F$ con $g l=18$ y 87 y $p<0,001)$. Al efectuar la reclasificación de los sexos por la mayor probabilidad, el 92\% fue asignado en el sexo del propio grupo. En la Figura 4 se muestran las asociaciones de los sexos de cada grupo empleando el método de clasificación de Ward a partir de los valores $D^{2}$ (Lambda de Wilks $=0,018$ aprox. $\left.F_{90,426}=6,113, p<0,001\right)$. Los hombres selk'nam forman un grupo aparte $y$, en un segundo orden, se disponen las mujeres halakwulup. Un grupo compacto se configura entre selk'nam y yámana femeninos y entre yámana y halakwulup masculinos que presentan entre sí los menores valores $D^{2}$. Las estadísticas del análisis multivariado para diversas comparaciones entre

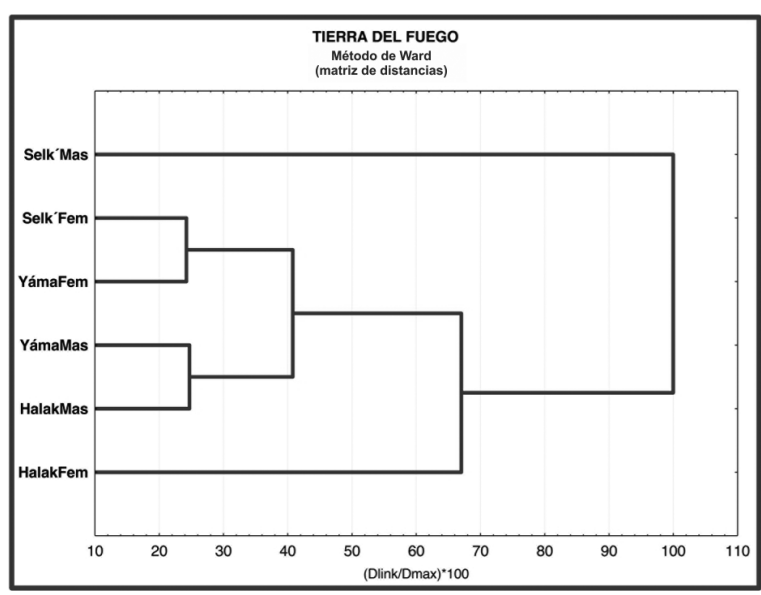

Figura 4. Asociación de grupos y sexos por valores $D^{2}$ y la técnica de clasificación de Ward. Selk: selk'nam, Yáma: yámana y Halak: halakwulup. Se agregan los sufijos Mas (masculinos) y Fem (femeninos) cuando corresponde. Lambda de Wilks: 0,018 aprox. $F_{90.426}=6,113, p<0,001$.

\begin{tabular}{|l|c|c|c|c|c|}
\hline \multirow{2}{*}{ Grupo } & \multirow{2}{*}{$\begin{array}{c}\text { Porcentaje } \\
\text { correcto }\end{array}$} & \multicolumn{3}{|c|}{ Grupo } & $\begin{array}{c}\text { Porcentaje } \\
\text { asignado } \\
\text { otro grupo }\end{array}$ \\
\cline { 3 - 5 } & & Selk'nam & Yamana & Halakwulup & 6,5 \\
\hline Selk'nam & 93,5 & 43 & 3 & 0 & 18,2 \\
\hline Yámana & 81,8 & 3 & 27 & 3 & 6,5 \\
\hline Halakwulup & 93,5 & 0 & 2 & 29 & $\mathbf{1 0 , 0}$ \\
\hline Total & $\mathbf{9 0 , 0}$ & $\mathbf{4 6}$ & $\mathbf{3 2}$ & $\mathbf{3 2}$ & \\
\hline
\end{tabular}

Tabla 4. Reclasificación de los individuos.

\begin{tabular}{|c|c|c|c|c|c|c|c|}
\hline & & \multicolumn{2}{|c|}{ Selk'nam } & \multicolumn{2}{|c|}{ Yámana } & \multicolumn{2}{|c|}{ Halakwulup } \\
\hline Grupos & Sexos & M & $\mathrm{F}$ & M & $\mathrm{F}$ & $M$ & $\mathrm{~F}$ \\
\hline \multirow{2}{*}{ Selk'nam } & M & 0,00 & 13,46 & 15,24 & 38,52 & 33,11 & 68,58 \\
\hline & $\mathrm{F}$ & 13,46 & 0,00 & 12,71 & 10,82 & 17,86 & 30,30 \\
\hline \multirow{2}{*}{ Yámana } & M & 15,24 & 12,71 & 0,00 & 15,92 & 11,03 & 36,86 \\
\hline & $\mathrm{F}$ & 38,52 & 10,82 & 15,92 & 0,00 & 11,75 & 12,34 \\
\hline \multirow{2}{*}{ Halakwulup } & M & 33,11 & 17,86 & 11,03 & 11,75 & 0,00 & 15,32 \\
\hline & $\mathrm{F}$ & 68,58 & 30,30 & 36,86 & 12,34 & 15,32 & 0,00 \\
\hline
\end{tabular}

Nota: M: masculino, F: Femenino. $D^{2}$ : Distancia de Mahalanobis (Prueba valores $F, g l=18$ y $87 ; p<0,001$ ).

Tabla 5. Valores $D^{2}$ entre grupos por sexo. 
grupos y entre sexos se presentan en la Tabla 6. En todos los casos, las diferencias son significativas.

De acuerdo con el modelo de equilibrio entre la deriva génica y la migración, se estimaron valores Fst para distintos casos, con la tasa migratoria $\mathrm{Nm}$ asociada. La divergencia entre etnias proporcionó el valor $F s t=0,170 \pm 0,010$ y una tasa migratoria de 1,22 ind-mig/gen (Tabla 7). En el análisis de los grupos de a pares se revelaron relaciones importantes que permiten evaluar la interacción entre etnias e inferir una estructura fenotípica territorial más precisa. Entre selk'nam-halakwulup se verifica el mayor valor $F s t=0,206 \pm 0,013$ con $N m=0,96$ ind-mig/gen; entre selk'nam-yámana, el valor $F_{s t}=0,100 \pm 0,011$ y $N m=2,24$ ind-mig/ gen; entre yámana-halakwulup, $F s t=0,089 \pm 0,011$ y $N m=2,56$ ind-mig/gen. Estos valores indican diferencias importantes en las relaciones entre grupos que permiten configurar dos circuitos de mayor interacción entre selk'nam-yámana y entre yámanahalakwulup (Tabla 7). Las varianzas residuales presentan valores significativos en todos los casos, a excepción de selk’nam (Tabla 8).

Considerando los grupos para cada sexo, se obtuvo un valor de $F s t=0,292 \pm 0,010$, con $N m=0,61$ ind-mig/gen (Tabla 7). Casi un 30\% de la varianza es producida por las diferencias entre los sexos de cada grupo. En la mayoría de los casos, la varianza residual presenta un valor de probabilidad significativo, excepto entre

\begin{tabular}{|l|c|c|c|c|}
\hline \multicolumn{1}{|c|}{ Entre } & $\begin{array}{c}\text { Lambda } \\
\text { de Wilks }\end{array}$ & $\boldsymbol{g} \boldsymbol{I}$ & aprox. F & $\boldsymbol{p}$ \\
\hline Grupos & 0,119 & 36 y 180 & 9,520 & $<0,0001$ \\
\hline Sexos-Grupos & 0,018 & 90 y 426 & 6,113 & $<0,001$ \\
\hline Masculinos-Grupos & 0,052 & 36 y 66 & 6,248 & $<0,0001$ \\
\hline Femeninos-Grupos & 0,052 & 36 y 74 & 6,996 & $<0,0001$ \\
\hline
\end{tabular}

Tabla 6. Evaluación de diferencias entre grupos y entre sexos.

\begin{tabular}{|l|l|l|l|}
\hline & Fst & e.e. & Nm \\
\hline Grupos & 0,170 & 0,010 & 1,22 \\
Selk'nam-Yámana & 0,100 & 0,011 & 2,24 \\
Selk'nam-Halakwulup & 0,206 & 0,013 & 0,96 \\
Yámana-Halakwulup & 0,089 & 0,011 & 2,56 \\
\hline Sexos-Grupos & 0,292 & 0,010 & 0,61 \\
Masculinos-Grupos & 0,260 & 0,015 & 0,71 \\
Femeninos-Grupos & 0,287 & 0,014 & 0,62 \\
\hline
\end{tabular}

Nota: e.e: error estándar, Nm: número de migrantes/generación.

Tabla 7. Valores Fst, errores estándar y migrantes por generación. masculinos yámana y halakwulup ( $p=0,114$ y $p=0,075$ respectivamente, Tabla 9). En la Figura 5, los grupos femeninos selk'nam y yámana presentan una varianza menor que la esperada por el equilibrio entre la deriva genética y la migración, producida por un menor tamaño efectivo. En cambio, se destaca la interacción en un amplio radio territorial por la mayor varianza y un mayor tamaño efectivo de las mujeres halakwulup y de los hombres selk'nam. Estos resultados se repiten pues las mujeres presentan valores Fst $=0,287 \pm 0,014$ y $N m=0,62$ ind-mig/gen; mientras que para los hombres se obtuvo Fst $=$ $0,260 \pm 0,015$ y $N m=0,71$ ind-mig/gen (Tabla 7). Excepto para la menor varianza residual de hombres selk'nam, los casos restantes presentan valores significativamente diferentes a los esperados (Tabla 9). Además, los hombres presentan una varianza generalizada entre grupos (determinante de la matriz de varianzas-covarianzas) diez veces mayor que la varianza de las mujeres $\left(\operatorname{Cov}{ }^{\lambda} / \operatorname{Cov} Q=5,65 E+29 / 5,67 E+28\right)$, quienes

\begin{tabular}{|l|l|l|l|l|l|}
\cline { 3 - 6 } \multicolumn{1}{|c|}{} & $\begin{array}{l}\text { Varianza } \\
\text { residual }\end{array}$ & $\begin{array}{l}\text { Error } \\
\text { estándar }\end{array}$ & Valor $\boldsymbol{t}$ & Valor $\boldsymbol{p}$ \\
\hline \multirow{6}{*}{ Grupos } & Selk'nam & $-0,031$ & 0,022 & 1,38 & $\mathbf{0 , 1 8 6}$ \\
& Yámana & $-0,128$ & 0,013 & 0,00 & 0,000 \\
& Halakwulup & 0,159 & 0,026 & 0,00 & 0,000 \\
\cline { 2 - 6 } & Selk'nam & $-0,049$ & 0,016 & 3,14 & 0,006 \\
& Yámana & 0,049 & 0,018 & 2,66 & 0,016 \\
\cline { 2 - 6 } & Selk'nam & $-0,099$ & 0,020 & 4,87 & 0,000 \\
& Halakwulup & 0,099 & 0,025 & 3,99 & 0,001 \\
\cline { 2 - 6 } & Yámana & $-0,062$ & 0,018 & 3,49 & 0,003 \\
& Halakwulup & 0,062 & 0,018 & 3,38 & 0,004 \\
\hline
\end{tabular}

Nota: Se destacan en negrita los valores de probabilidad no significativos. Tabla 8. Evaluación de la varianza residual, error estándar, valores $t$ y de probabilidad $p$. Etnias en conjunto y de a pares.

\begin{tabular}{|l|l|l|l|l|l|}
\cline { 3 - 6 } \multicolumn{2}{l|}{} & $\begin{array}{l}\text { Varianza } \\
\text { residual }\end{array}$ & $\begin{array}{l}\text { Error } \\
\text { estándar }\end{array}$ & Valor $\boldsymbol{t}$ & Valor $\boldsymbol{p}$ \\
\hline \multirow{2}{*}{ Selk'nam } & Masculinos & 0,147 & 0,045 & 3,30 & 0,004 \\
& Femeninos & $-0,171$ & 0,023 & 7,42 & 0,000 \\
\hline \multirow{2}{*}{ Yámana } & Masculinos & $-0,053$ & 0,032 & 1,67 & $\mathbf{0 , 1 1 4}$ \\
& Femeninos & $-0,159$ & 0,026 & 6,06 & 0,000 \\
\hline \multirow{3}{*}{ Halakwulup } & Masculinos & 0,057 & 0,030 & 1,89 & $\mathbf{0 , 0 7 5}$ \\
& Femeninos & 0,179 & 0,053 & 3,37 & 0,004 \\
\hline \multirow{3}{*}{ Femeninos } & Selk'nam & $-0,073$ & 0,034 & 2,13 & $\mathbf{0 , 0 4 8}$ \\
& Yámana & $-0,166$ & 0,025 & 6,65 & 0,000 \\
& Halakwulup & 0,239 & 0,046 & 5,21 & 0,000 \\
\hline & Selk'nam & 0,112 & 0,040 & 2,82 & 0,012 \\
& Halmana & $-0,217$ & 0,017 & 12,59 & 0,000 \\
\hline
\end{tabular}

Nota: Se destacan en negrita los valores de probabilidad no significativos.

Tabla 9. Evaluación de la varianza residual, error estándar, valores $t \mathrm{y}$ de probabilidad $p$. Análisis por sexo y grupos. 


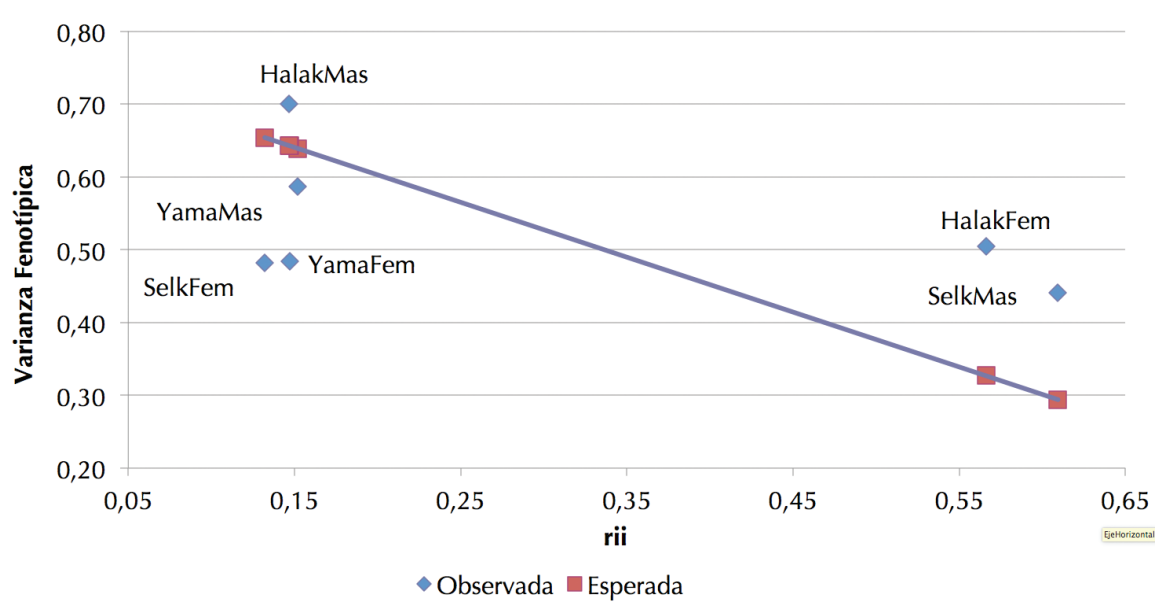

Figura 5. Distribución de la varianza fenotípica observada (rombos) y esperada (cuadrados) entre sexos de selk'nam (Selk), yámana (Yama) y halakwulup (Halak). Se agregan los sufijos Mas (masculinos) y Fem (femeninos) cuando corresponde. Valores rii: distancia al centroide de la distribución. Valor $F s t=0,292 \pm 0,010$.

poseen mayor homogeneidad genética dentro de grupos y un menor tamaño efectivo asociado con un circuito de relaciones más restringido. Esta evidencia sugiere un patrón de residencia matrilocal.

\section{DISCUSIÓN}

En la muestra obtenida por Gusinde (1989 [1937]) se observa un equilibrio entre la distribución de los sexos y de las edades. Hay un $8 \%$ más de mujeres, aunque las diferencias estadísticas no son significativas (Tabla 2). Tampoco existen pruebas suficientes para detectar diferencias en la distribución por edades entre los grupos (Tabla 3), aunque predominan los individuos adultos jóvenes de 21-30 años (Figura 2). En general, las diferencias entre los fenotipos de las etnias son significativas de acuerdo con las pruebas $\mathrm{D}^{2}$ y el análisis discriminante. También es consistente la prueba de reclasificación (Tabla 4): un 90\% de casos fueron asignados al propio grupo. Yámana presenta más casos reasignados a selk'nam y halakwulup en un $18 \%$. Estos resultados son semejantes a los obtenidos por Varela et al. (1993-1994) y marcan el efecto de la distancia espacial entre los grupos. Además, las determinaciones concuerdan con las actuales pruebas sobre las diferencias estadísticas para varios diseños: entre grupos, entre grupos para cada sexo y entre sexos de cada grupo (Tabla 6).

La evaluación fenotípica del dimorfismo sexual en cada etnia (Tabla 5) es consistente con la reclasificación de los individuos, pues solamente el $8 \%$ de los casos fue asignado a un grupo diferente del original. Se observó una particular estructura que refleja relaciones de parentesco distintas entre las etnias en general y en particular entre los sexos. Aunque las pruebas estadísticas fueron significativas en todas las comparaciones, sobre la base de las distancias fenotípicas $\left(D^{2}\right)$, los hombres selk'nam y las mujeres halakwulup se diferencian del conjunto; mientras que se destacaron dos asociaciones: una entre las mujeres selk'nam y yámana, y otra entre los hombres yámana y halakwulup (Figura 4).

De acuerdo con la evaluación del modelo de equilibrio entre deriva/migración (Relethford y Harpending 1994), existen relaciones entre las etnias y entre las etnias por sexos, coherentes con la distribución geográfica consignada por Gusinde (1989 [1937]). En el primer caso, con el 17\% de variación entre grupos, la desviación del valor de varianza esperado para halakwulup indica el efecto de un flujo génico externo desde un espacio territorial mayor (canales de Patagonia Occidental, islas e islotes entre la península Brecknock, entre otros), tal como anotara Gusinde (1989 [1937]). En cambio, yámana refleja un circuito de actividad más restringido en el archipiélago del cabo de Hornos y la costa norte del canal de Beagle por la menor varianza fenotípica y un posible tamaño efectivo reducido. El grupo selk'nam se encuentra más próximo al equilibrio entre los efectos de la deriva génica y de la migración por un balance diferente entre las propiedades de hombres y mujeres (Figura 5 y Tabla 9).

Las relaciones entre pares de grupos revelaron los niveles de interacción más probables (Tabla 7) y permiten reconstruir las relaciones posibles entre las etnias. Por ejemplo, fueron más probables las relaciones entre selk'nam-yámana, con un $10 \%$ de varianza fenotípica entre grupos y un nivel migratorio de 2,24 ind-mig/gen; y entre yámana-halakwulup, cuya varianza entre grupos alcanza a $9 \%$ y 2,56 ind-mig/gen. En cambio, entre selk'nam-halakwulup las relaciones son menos frecuentes, pues la varianza entre etnias es de $21 \%$ con 0,96 ind-mig/ gen. Además, el flujo génico externo para ambas etnias se relaciona con territorios distintos. Las distancias $D^{2}$ y la reclasificación de los individuos son 
consistentes con estos resultados, pues los mayores valores se dan entre selk'nam y halakwulup (Figura 3) y es nula la reclasificación de los individuos entre ambos grupos (Tabla 4). Es interesante destacar la proximidad del valor Fst entre yámana-halakwulup ( $F s t=0,089 \pm 0,011)$, estimado en este trabajo con variables fenotípicas, y los calculados con mtDNA por Saint-Pierre et al. (2012) para muestras actuales $(F s t=0,112, p=0,019)$ y los obtenidos por De la Fuente et al. (2015) con muestras actuales $(F s t=0,107, \mathrm{p}=0,004)$ y antiguas ( $F s t=0,129$, $\mathrm{p}<0,001)$. Además, con variables craneométricas se establecieron relaciones entre un conjunto de muestras desde el río Deseado hasta el extremo continental austral (Cocilovo y Guichón 1985-1986, 1999-2000), una experiencia que debería de ser actualizada en el futuro.

Se estableció una estructura poblacional importante a partir de la experiencia realizada entre grupos por sexos, en la cual los valores Fst muestran la combinación de la varianza entre grupos máxima con la varianza dentro de grupos mínima y un mayor nivel de parentesco local (Tabla 7), que refleja la conservación de la mayor variabilidad genética dentro de los sexos de cada etnia. Además, la varianza generalizada entre grupos del sexo masculino -diez veces mayor que la del sexo femenino- tal vez esté asociada con una posible pauta de residencia matrilocal relacionada con un circuito territorial más restringido. Esta inferencia es consistente con los mayores valores $D^{2}$ entre los hombres de cada grupo con relación a los valores de las mujeres (Tabla 5). También se puede establecer un circuito de relaciones más probable por las afinidades entre selk'nam y yámana y entre yámana y halakwulup.

Las observaciones consignadas en la obra de Gusinde revelan indicios que permiten explicar los resultados de la estimación del tamaño efectivo, de la migración y del apareamiento dentro del grupo o entre grupos locales y foráneos. Por ejemplo, en selk'nam se consignan dos casos de parentesco filial (madre-hija y dos hermanas) y se incluye un individuo haus (Tomo 4: 30-31); se menciona la práctica exogámica con la prohibición del matrimonio dentro de la misma localidad o grupo familiar, pero se habilita entre grupos más distantes (Tomo 4: 65). Con relación a yámana, se declaran varios individuos emparentados directamente (progenitor descendiente), individuos de padre yámana y madres selk'nam y halakwulup (Tomo 4: 76-77) y práctica exogámica local (Tomo 4: 111), aunque no se descartan parentescos consanguíneos. Para halakwulup, integrado con individuos de un amplio territorio, se mencionan relaciones de parentesco filial y en segundo grado, así como apareamientos con miembros de las otras etnias locales y con europeos. Se destaca el impacto en la población por el contacto con europeos y el contagio de enfermedades venéreas (Tomo 4: 122, 123 y 159).

De acuerdo con el modelo de Relethford y Harpending (1994), los sexos manifiestan un comportamiento distinto, que permite explicar el resultado anterior (Figura 5). Los hombres selk'nam y las mujeres halakwulup presentan una mayor varianza fenotípica con una menor variabilidad dentro de grupo por la interacción en un amplio territorio y el efecto de un flujo génico externo. En el caso de las mujeres halakwulup, se incluyen relaciones consensuadas o forzadas con europeos. Los hombres y las mujeres yámana confirman la observación realizada sobre sus relaciones en un ámbito local con un menor tamaño efectivo y una mayor interacción entre asentamientos próximos, de acuerdo con los comentarios del párrafo anterior tomado de Gusinde (1989 [1937]). La varianza de las mujeres selk'nam es mucho menor que la esperada y muy próxima a las mujeres yámana con las cuales existen relaciones de parentesco, circunstancia que explica su posición en la Figura 5. La tasa migratoria general $(\mathrm{Nm}=1,22$ ind-mig/gen $)$ podría compensar teóricamente el efecto de la deriva genética; sin embargo, cuando este valor se estima entre grupos para cada sexo, su efecto disminuye $(\mathrm{Nm}=0,71$ ind-mig/gen en el caso de hombres $y$ $\mathrm{Nm}=0,62$ ind-mig/gen en el caso de mujeres), lo cual sustenta una moderada divergencia genotípica entre los sexos de las distintas etnias.

Los resultados obtenidos a partir del estudio de las etnias selk'nam, yámana y halakwulup son consistentes con los análisis matrilineal y patrilineal a partir de haplogrupos mitocondriales (mtDNA) y del cromosoma Y. En todos los casos, se destaca la menor variación genética en el extremo sur continental de los habitantes de Tierra del Fuego por los efectos del aislamiento territorial y la pérdida de variabilidad genética por distintos mecanismos evolutivos: migración, deriva genética, efecto fundador, entre otros (Lalueza et al. 1997; García et al. 2004; García-Bour et al. 2004; Moraga et al. 2010; De Saint Pierre et al. 2012; De la Fuente et al. 2015). Dichos factores influyeron en la estructura de la población, en el tamaño efectivo, en el tamaño de la familia y en la supervivencia de la población, hasta la cruda fase final de la extinción por el impacto de la conquista y colonización europea. 
En síntesis, los resultados permiten sustentar la puesta en valor de la obra de Gusinde (1989 [1937]) que aún hoy conserva y permite proporcionar una información valiosa para evaluar las condiciones de vida y la supervivencia de las poblaciones que colonizaron un territorio inhóspito en los confines de la ecúmene. Se comprobaron las diferencias físicas entre las etnias, las características del dimorfismo sexual, las relaciones de parentesco y el proceso de divergencia genética; se evaluaron los efectos de la deriva génica y la migración en general y en particular, entre grupos y entre grupos por sexo. Además, se infiere la existencia de circuitos y territorios de interacción más probables y la posible pauta de residencia matrilocal. La mayor variabilidad genética se conservaba en los sexos de cada etnia.

\section{Agradecimientos}

La realización del presente trabajo fue posible gracias al apoyo brindado por el Consejo Nacional de Investigaciones Científicas y Técnicas (PIP N ${ }^{\circ}$ 112201101 00833) y la Secretaría de Ciencia y Técnica, Universidad Nacional de Río Cuarto (SeCyT-UNRC, 852/11 y 161/16). Agradecemos también a los revisores por las sugerencias realizadas, que contribuyeron a su mejoramiento.

\section{REFERENCIAS CITADAS}

Casali, R.

2013 Movilidad y uso del espacio: análisis demográfico de la trayectoria selk'nam ante la colonización. Tierra del Fuego, Argentina, 1890-1930. Anuario del Instituto de Historia Argentina 13: 1-18.

Cocilovo, J. A. y R. A. Guichón

1985-1986 Propuesta para el estudio de las poblaciones aborígenes del extremo austral de Patagonia. Anales Instituto de la Patagonia (Serie Ciencias Sociales) 16: 111-123.

1999-2000 La variación geográfica y el proceso de microdiferenciación de las poblaciones aborígenes de Patagonia Austral y de Tierra del Fuego. Revista Chilena de Antropología 15: 9-28.

De La Fuente, C., J. Galimany, B. M. Kemp, K. Judd, O. Reyes y M. Moraga

2015 Ancient Marine Hunter-Gatherers from Patagonia and Tierra del Fuego: Diversity and Differentiation Using Uniparentally Inherited Genetic Markers. American Journal of Physical Anthropology 158 (4):
719-729. doi: 10.1002/ajpa.22815

De Saint Pierre, M., C. M. Bravi, J. M. B. Motti, N. Fuku,

M. Tanaka, E. Llop, S. L. Bonatto y M. Moraga

2012 An Alternative Model for the Early Peopling of

Southern South America Revealed by Analyses of Three

Mitochondrial DNA Haplogroups. PLOS ONE 7 (9):

e43486. doi: 10.1371/journal.pone.0043486

Falconer, D. S. y T. F. C. Mackay

1996 Introduction to Quantitative Genetics. Longman, Essex.

García, F., M. Moraga, S. Vera, H. Henríquez, E. Llop, C.

Ocampo, E. Aspillaga y F. Rothhammer

2004 Origen y microdiferenciación de la población humana del archipiélago de Chiloé. Revista Chilena de Historia Natural 77: 539-546.

García-Bour, J., A. Pérez-Pérez, S. Álvarez, E. Fernández,

A. M. López-Parra, E. Arroyo-Pardo y D. Turbón 2004 Early Population Differentiation in Extinct Aborigines from Tierra del Fuego-Patagonia: Ancient mtDNA Sequences and Y-Chromosome STR Characterization. American Journal of Physical Anthropology 123 (4): 361 370. doi: 10.1002/ajpa.10337

García-Moro, C., M. Hernández y C. Lalueza 1997 Estimation of the Optimum Density of the Selk'nam from Tierra del Fuego: Inferences about Human Dynamics in Extreme Environments. American Journal of Human Biology 9: 699-708. doi: 10.1002/ (SICI)1520-6300(1997)9:6<699::AID-AJHB3>3.0.CO;2-N

Guichón, R. A., I. Martí, E. Aspillaga, J. A. Cocilovo y F. Rothhamer

1989-1990 Contribución al conocimiento de las relaciones biológicas entre las poblaciones aborígenes de Patagonia austral y Tierra del Fuego. Runa 19: 27-39.

Gusinde, M 1989 [1937] Los indios de Tierra del Fuego, Antropología Física, Tomo IV, Vol. 1. Centro Argentino de Etnología Americana, Buenos Aires.

Lalueza, C., A. Pérez-Pérez, E. Prats, L. Cornudella y D. Turbón 1997 Lack of Founding Amerindian Mitochondrial DNA Lineages in Extinct Aborigines from Tierra del FuegoPatagonia. Human Molecular Genetics 6 (1): 41-46. doi: 10.1093/hmg/6.1.41

Medeot, E. A., J. A. Cocilovo y H. H. Varela 2008 Varianza Ambiental y Estabilidad del Desarrollo en la Población Prehispánica de Las Pirguas (Salta, Argentina). Latin American Antiquity 19 (2): 146-157. doi: $10.1017 /$ S1045663500007768 
Miotti, L. L. y M. C. Salemme

2004 Poblamiento, movilidad y territorios entre las sociedades cazadoras-recolectoras de Patagonia. Complutum 15: 177-206.

Montes Suay, F.

2007 Introducción a la probabilidad. Departamento de Estadística e Investigación Operativa, Universitat de València, Burjassot, España.

Moraga, M. L., M. De Saint Pierre, F. Torres y J. Ríos 2010 Vínculos de parentesco por vía materna entre los últimos descendientes de la etnia kawésqar y algunos entierros en los canales patagónicos: evidencia desde el estudio de linajes mitocondriales. Magallania 38 (2): 103-114. doi: 10.4067/S0718-22442010000200006

Morello F., L. A. Borrero, J. Torres, M. Massone, M. Arroyo, R. Mcculloch, E. Calás, M. Lucero, I. Martínez y G. Bahamonde 2009 Evaluando el registro arqueológico de Tierra del Fuego durante el Holoceno temprano y medio. En Arqueología de la Patagonia: una mirada desde el último confín, editado por M. C. Salemme, F. Santiago, M. Álvarez, E. Piana, M. Vázquez y M. E. Mansur, pp. 1075-1091. Utopías, Ushuaia, Tierra del Fuego.

Morello, F., L. A. Borrero, M. Massone, C. Stern, A. García-Herbst, R. McCulloch, M. Arroyo-Kalin, E. Calás, J.

Torres, A. Prieto, I. Martínez, G. Bahamonde y P. Cárdenas 2012 Hunter-gatherers, Biogeographic barriers and the development of human settlement in Tierra del Fuego. Antiquity 86: 71-87. doi: 10.1017/S0003598X00062463

Ocampo, E. C. y P. Rivas H.

2004 Poblamiento temprano de los extremos geográficos de los canales patagónicos: Chiloé e isla Navarino, Simposio Ocupaciones Iniciales de Cazadores Recolectores en el sur de Chile (Fuego-Patagonia y Araucanía). Chungara, Revista de Antropología Chilena Volumen Especial: 317-331. doi: 10.4067/ S0717-73562004000300034
Relethford, J. H.

1994 Craniometric Variation among Modern Human Populations. American Journal of Physical Anthropology 95 (1): 53-62. doi: 10.1002/ajpa.1330950105

\section{RMET}

2003 RMET 5.0 Programa. http://employees.oneonta.edu/ relethjh/programs/

Relethford, J. H. y J. Blangero

1990 Detection of Differential Gene Flow Patterns of Quantitative Variation. Human Biology 62: 5-25.

Relethford, J. H., M. H. Crawford y J. Blangero 1997 Genetic Drift and Gene Flow in Post-Famine Ireland. Human Biology 69: 443-465.

Relethford, J. H. y H. C. Harpending

1994 Craniometric variation, genetic theory, and modern human origins. American Journal of Physical Anthropology 95 (3): 249-270. doi: 10.1002/ ajpa.1330950302

Seber, G. A. F.

1984 Multivariate Observations. John Wiley and Sons, Nueva York.

Varela, H. H. y J. A. Cocilovo

1999 Evaluation of the Environmental Component of the Phenotypic Variance in Prehistoric Populations. HOMO 50 (1): 46-53.

2007 Phenotypic, maximum genetic, and special environmental variability in prehistoric human populations. American Journal of Physical Anthropology 132 (1): 17-24. doi: 10.1002/ajpa.20473

Varela, H. H., J. A. Cocilovo y R. A. Guichón 1993-1994 Evaluación de la información somatométrica publicada por Gusinde sobre los aborígenes de Tierra del Fuego. Anales Instituto de la Patagonia, Serie Ciencias Históricas 22: 193-205.

1997 Evaluation of the craniometric information published by Gusinde about the natives from Tierra del Fuego. HOMO 48 (2): 125-134. 